\title{
Las políticas de proyección social universitaria en Perú: Realidad y desafíos
}

Efraín Fernando Miranda Suárez

emiranda@uncp.edu.pe

Evita Marisol Fernández Villanueva emarisolfv@hotmail.com

Luís Alberto Miranda Chávez

Luismicha1793@gmail.com

David Raúl Hurtado Tiza

Dahutiepg7@ hotmail.com

Universidad Nacional del Centro del Perú

\section{RESUMEN}

Las universidades en el Perú, son las formadoras de cuadros profesionales para la sociedad y las empresas, y aun estando preocupados por la calidad educativa para su inserción laboral han desatendido el compromiso de ser orientadores del desarrollo del ámbito de su influencia. Este estudio tiene como objetivo analizar las características que presentan las políticas de proyección social en las universidades peruanas, determinando el papel de la proyección social universitaria en el nuevo contexto peruano y comparar la relación de la proyección social/extensión universitaria con los procesos de formación e investigación. Es un estudio mixto metodológicamente, se realiza en la macro región central del Perú en 13 universidades: 10 públicas y 3 privadas. La información es recogida por un cuestionario adaptado sobre la base de las metas e indicadores de RSU en participación social diseñado para la URSULA por Vallaeys y Solano (2018). Se aplicó vía correo electrónico a dos informantes clave por cada una. Se complementó la investigación con una lista de cotejo y el análisis documental de los instrumentos de gestión universitaria. El análisis de los datos estructurados concluye que la Proyección Social como la tercera función fundamental en las universidades del Perú no cuenta con políticas públicas específicas, como si lo tienen otros países de América Latina. La ausencia de políticas de proyección social ha propiciado una débil articulación con los procesos de formación e investigación con dinámicas aisladas lo que dificulta la formación integral del estudiante e interfiere el cumplimiento del compromiso histórico 
de la universidad con la sociedad. El estudio devela también que la función de proyección social resulta devaluada al considerarse como tarea "secundarias", por lo mismo la universidad no participa en la agenda pública y no puede promover el desarrollo territorial de su ámbito de influencia.

Palabras Clave: Proyección/extensión universitaria; Política universitaria; Formación académica, Investigación; RSU, Planeamiento Estratégico 


\title{
University social projection policies in Peru: Reality and challenges
}

\begin{abstract}
The universities in Peru are the trainers of professional cadres for society and companies, and even being concerned about the educational quality for their labor insertion, they have neglected the commitment to be guidance for the development of the sphere of their influence. This study aims to analyze the characteristics of social projection policies in Peruvian universities, determining the role of university social projection in the new Peruvian context and to compare the relationship of social projection / university extension with the processes of training and education. Investigation. It is a methodologically mixed study, it is carried out in the central macro region of Peru in 13 universities: 10 publics and 3 privates. The information is collected by an adapted questionnaire based on the goals and indicators of RSU in social participation designed for URSULA by Vallaeys and Solano (2018). It was applied via email to two key informants for each one. The research was complemented with a checklist and documentary analysis of the university management instruments. The analysis of the structured data concludes that Social Projection as the third fundamental function in Peruvian universities do not have specific public policies, as do other Latin American countries. The absence of social projection policies has led to a weak articulation with the training and research processes with isolated dynamics, which hinders the comprehensive training of the student and interferes with the fulfillment of the university's historical commitment to society. The study also reveals that the function of social projection is devalued as it is considered as a "secondary" task, therefore the university does not participate in the public agenda and cannot promote the territorial development of its sphere of influence.
\end{abstract}

Key words: University outreach / extension; University policy; Academic training; Research, USR; Strategic Planning

Artículo recibido: 05 de Abril 2021 Aceptado para publicación: 28 de Mayo 2021 Correspondencia: emiranda@uncp.edu.pe Conflictos de Interés: Ninguna que declarar 


\section{INTRODUCCIÓN}

Para los efectos de este estudio se entiende por políticas de proyección social o extensión universitaria al conjunto de normativas que tiene una institución universitaria para cumplir su función de relacionamiento con el territorio de su influencia y sus requerimientos de manera sostenible, formas de relacionar la educación universitaria, la cultura y la sociedad para generar un "diálogo de saberes" en términos de Tommassino (2017) o como "ecología de saberes" según Sousa Santos (2012). Debe estar alineada a la visión, misión y objetivos estratégicos que los distingue orientando las acciones que deben cumplir en esta función sustantiva, su articulación con las otras funciones: formación académica e investigación. Señalará las estrategias para interactuar en la sociedad y sus grupos de interés, demarcando sus alcances y límites para evitar confusión con otras acciones que no son de la naturaleza de extensión universitaria u otras que al no estar incluidas en las normas institucionales vienen a ser "actividades informales", "residuales" o "difuso" de la extensión universitaria como advierte Menéndez (2018). Para evitar ello hay necesidad de contar con políticas institucionales que orienten sus funciones, en tanto que, según Mintzberg y Jorgensen, (1995) las “políticas”, consisten en lineamientos generales, que constituyen el "paraguas" bajo el cual otros actores formularán las estrategias específicas. Vale decir que es actuar estratégicamente u "obrar en función a fines" como lo señalaría Weber (1977) o en términos de Habermas (1987) con "racionalidad instrumental", entendida como la acción que se ajusta a reglas técnicas para causar efectos positivos esperados en el estado de cosas y sucesos, en este caso en la extensión o proyección social universitaria.

Resulta entonces, primordial conocer cuáles son las políticas de proyección social en las universidades del Perú, qué modelos han perfilado, cómo responden a los retos de la globalización, la inviabilidad del planeta, la realidad socio económica cultural del país, cómo orientan su cumplimiento en el interno y en el externo, de qué manera se establece el relacionamiento con su entorno, cómo está integrado a las otras funciones de la universidad y cómo se financia estos proyectos de intervención social. Todo esto podrá tener respuesta si se tienen políticas de extensión universitaria establecidas institucionalmente. Para abordar estas inquietudes hay necesidad de hacer un recorrido del proceso que ha seguido esta función universitaria. 
En el continente europeo el primer programa de Extensión nació en la Universidad de Cambridge en 1867 (Núñez, Álvarez y Martínez ,2017; Menéndez 2018) con el antecedente de los Colegios de la Clase Obrera de Sheffield que tuvo como propósito ofrecer educación a jóvenes de la clase trabajadora, luego se fue expandiendo a otras universidades europeas: Alemania, Hungría, Rusia y Francia con sus famosas “universidades Populares”. En América se considera que las actividades de extensión universitaria en las universidades liberales de entonces a partir de la instauración de los Land Grant Colleges en 1862, impartiendo lecciones de mecánica y agricultura, el propósito era expandir la educación superior a la mayoría de la población y establecer relaciones con el entorno inmediato y la sociedad en general.

Las universidades de América Latina y el Perú rompen con el modelo aristocrático elitista Influenciados por el Manifiesto de Córdoba Argentina (1918) pasando a adquirir nuevos compromisos. La Carta de Liminar que impulsa la primera Reforma universitaria se extiende rápidamente en el continente y entre sus varios postulados destaca el compromiso social de la universidad con el cambio de la sociedad, la solidaridad y diálogo con el pueblo comprometiéndolo a trasuntar sus fronteras para ponerse al servicio de la sociedad y su problemática. Naciendo de esta manera la extensión universitaria como la tercera función fundamental del sistema universitario junto a la formación profesional y la investigación (Tünnermann: 2002, 2008; Fernández: 2014).

El propósito y conceptualización de la extensión universitaria pasó por un proceso que va desde el Primer Congreso de Universidades Latinoamericanas de Guatemala en 1949 hasta la adopción de la Responsabilidad Social Universitaria (RSU) con la experiencia del Proyecto Construye País en Chile el 2000. En estos cincuenta años el concepto sufre modificaciones desde la Carta de las universidades latinoamericanas de la Unión de Universidades de América Latina (UDUAL) que organizó la Primera Conferencia Latinoamericana de Extensión Universitaria y Difusión Cultural en Santiago de Chile en 1957 donde se definió el primer concepto de extensión universitaria como " misión y función orientadora de la universidad contemporánea (...) que tiene por misión proyectar, en la forma más amplia posible y en todas las esferas de la nación, los conocimientos, estudios e investigaciones de la universidad, para permitir a todos participar en la cultura universitaria, contribuir al desarrollo social y a la elevación del nivel espiritual, moral, intelectual y técnico". La Segunda Conferencia latinoamericana de Extensión 
universitaria y difusión cultural realizada en México en 1972 donde se redefine como "la interacción de la universidad con los demás componentes del cuerpo social, a través de la cual ésta asume y cumple su compromiso de participación en el proceso de creación de la cultura y de liberación y transformación radical de la comunidad nacional" (D'Andrea, R. E., Zubiría, A. y Sastre Vázquez, P. (s/f). En 1998, la Conferencia Mundial sobre Educación Superior invita a la universidad a "a ser más solidaria con el servicio a la comunidad, colaborar a erradicar la pobreza, la intolerancia, la violencia, el analfabetismo, el hambre y la sostenibilidad del medio ambiente". Finalmente, La Conferencia Mundial de Educación Superior realizada en París el 2009, introduce el tema de responsabilidad social como marco de la actividad universitaria.

De las tres fundamentales funciones de la universidad peruana y latinoamericana, la extensión universitaria es la que ha experimentado mayores retos de reconfiguración para responder a las coyunturas que le ha tocado vivir. Así la "misión social" de la universidad denominada inicialmente como "extensión universitaria" adquiere en adelante otras denominaciones tales como: "proyección social", "relaciones con la comunidad", "extensión Interactiva", "labor de extensión", "relación universidad sociedad", "compromiso social" "servicio comunal universitario", "trabajo social universitario", "servicio social", "servicio a la comunidad", "acción social", "vinculación con la sociedad", "vinculación con el medio" entre otros. En ésta dinámica, sus labores han ido tomando diversos perfiles y modalidades cambiando constantemente sus propósitos y funciones desde el compartimiento del conocimiento académico con el entorno mediante: trasferencias de tecnologías, difusión de información, capacitación, asesorías y consultorías, hasta el servicio cultural - artístico pasado por asistencialismo filantrópico con el "voluntariado universitario" y prácticas deportivas entre otras. Por su puesto que tras de cada una de ellas hay concepciones ideológico políticas imperantes (Menéndez 2018).

La relación universidad -sociedad fue asimétrica y unidireccional en sus primeros momentos y fue mutando a formas más horizontales e interactivas de los actores participantes en la detección de la problemática y necesidades para el caso argentino según señala Gómez (2019); en el Perú ,fue algo similar hasta los años 80 del siglo anterior con un acercamiento cada vez más fuerte a las comunidades y sectores vulnerables, pero la violencia política trastocó la relación de la universidad con la 
sociedad, la mayoría de universidades se vieron obligadas a trabajar dentro de sus recintos con tareas de: implementación de ambientes, imagen institucional, publicación de revistas y libros, asesoría académica, arreglos ambientales del campus universitario, cultivo y difusión de expresiones artístico culturales, cine, museos etc. Después de la pacificación se produjo un tímido acercamiento de la universidad a las comunidades y sectores vulnerables (Miranda-Suárez 2010) lo que ha trastocado las formas y propósitos organizativos. Las universidades privadas enrumbaron sus relaciones con las empresas, organismos internacionales y otras que dio origen a formas de extensión universitaria con: acciones de prácticas, intercambio de experiencias, pasantías entre otras, mientras que las universidades nacionales retomaron sus formas anteriores con muchas dificultades y sin financiamiento, lo que condujo a distorsiones en la conceptualización, la teleología y ejecución.

Las políticas públicas sobre Extensión universitaria en el Perú son escasas y poco claras lo que originó políticas institucionales diferentes y hasta contradictorias entre sí. La Ley Universitaria $\mathrm{N}^{\circ} 13417$ del año 1960 introduce por primera vez el término de Extensión Universitaria, pero con una imprecisión conceptual, lo que origina distintas interpretaciones que cada universidad le da. La Ley Universitaria $\mathrm{N}^{\circ} 23733$ promulgada en 1983 y vigente hasta el 2014, denomina y reconoce a la "Proyección y Extensión Universitaria" como una de las funciones sustantivas de la Universidad junto a las de formación profesional e investigación, el Capítulo IX: De la Extensión y Proyección Universitaria en su artículo 68 indica: "las Universidades extienden su acción educativa a favor de quienes no son estudiantes regulares y da asistencia". Como se observa, la Ley no hace una clara distinción entre la denominada "Proyección Social" y la "Extensión Universitaria", lo cual da origen a distintas interpretaciones en las universidades peruanas. Ante tal situación el 2007 la Asamblea Nacional de Rectores (ANR) preocupada por encontrar una mejora de éstas actividades, solicitó información a 91 universidades sobre la denominación con el que regulaban esta función, teniendo como resultado que solo contestaron oficialmente 22 de ellas el $70 \%$ lo hacían como proyección universitaria y el $30 \%$ como extensión universitaria. Complementariamente la ANR tomó una muestra de 10 universidades latinoamericanas, encontrando en ellas que el $65 \%$ lo enfocan como extensión universitaria y el 35\% como proyección universitaria (CONEAU 2008).

Ciencia Latina Revista Científica Multidisciplinar, Ciudad de México, México. 
Con la promulgación de la Nueva Ley Universitaria $N^{\circ} 30220$ del 2014 que incluye en su Título XIII, Artículos 24 y 25 la Responsabilidad Social Universitaria (RSU) como compromiso para el desarrollo sostenible, la relación de la universidad con la sociedad se ve trastocada. Cada universidad empezó a adoptarla indistintamente suscitando un variopinto de formas del relacionamiento universidad - sociedad. Unas reemplazaron la extensión universitaria o proyección social por el de RSU, Vallaeys (2016) advierte que "en la última década, muchas universidades han rebautizado sus áreas de vinculación social en términos de RSU” (p.10) corroborado por Miranda-Suárez (2018), otras las fusionaron y algunas las juntaron con el bienestar social universitario; unos le dieron la orientación por el trabajo en valores, otros en asuntos medioambientales, de salud o priorización del voluntariado, entre otras formas, de manera que la histórica y tercera función fundamental de la universidad se ve distorsionada. El sistema educativo universitario ha priorizado la calidad de la formación profesional y la investigación, pero ha dejado de lado la extensión universitaria lo cual es preocupante en tanto que la formación debe ser integral y la extensión universitaria es la vía de hacerla. Todo esto se configura a partir de que la Ley 30220 no ha definido las funciones de la proyección social como sí lo tenía la anterior ley.

La adopción de la RSU en las universidades peruanas es legalmente de carácter obligatorio como lo señala la ley y no es optativa o voluntaria como en otros países. Esta situación hace que en adelante toda universidad debe de ser socialmente responsable, lo que implica cambios sustantivos en su cultura organizacional y las consecuentes modificaciones epistémico cognitivas en sus procesos y funciones comprometiendo a toda la comunidad universitaria. Es decir, no es función de una oficina o una comisión, es un compromiso y accionar de todos cuantos viven en la universidad en el estatus y rol que tienen como actores. En este orden de ideas el contexto de la educación universitaria en América Latina de acuerdo a (Gargantini 2014) y particularmente en el Perú se encuentra actualmente tensionado por una serie de exigencias que exigen el replanteamiento de sus funciones y preponderantemente su misión frente a la sociedad y la realidad actual.

Siendo así, el concepto de "compromiso social" de la universidad se ve trastocado por el de "responsabilidad social" en tanto que "como responsabilidad es más radical y exigente que un compromiso, porque es anterior a cualquier voluntad de actuar" según señala 
Vallaeys (2019) es decir se convierte de este modo en "un deber" ineludible y fundamental que compromete la ética en toda la actividad y cotidianidad del quehacer universitario.

En medio de este estado situacional este estudio tiene como objetivo: Sistematizar las características que presentan las políticas de proyección social en las universidades peruanas, determinar el papel de la proyección social universitaria en el nuevo contexto peruano y comparar la relación de la proyección social/extensión universitaria con los procesos de formación e investigación. El estudio se justifica porque incita a reflexionar sobre el quehacer de la proyección social / extensión universitaria en medio de la adopción de la RSU en las universidades del Perú, así mismo aportar a la construcción de una teoría sobre el tema de proyección social universitaria, metodológicamente el trabajo pudo validar un cuestionario que da cuenta de los indicadores de la proyección social como variable a ser observado y medido y en términos prácticos los resultados de la investigación pueden ser utilizados como insumo para la formulación de las políticas de proyección social universitaria.

La producción de estudios sobre este tema es escasa, insuficientes para tener un estado del arte. Las investigaciones sobre la problemática universitaria al parecer no le han dado importancia a la función de proyección social. Sin embargo, hay estudios que en algo dan cuenta y ayudan en su abordamiento:

Cano, A. y Castro, D. (2016) en su trabajo La extensión universitaria en la transformación de la Educación superior, el caso Uruguay. Refiere que la Universidad de la República (ULAR) tiene políticas expresadas en una Resolución para desarrollar esta labor aprobado por su Consejo Directivo, a partir del cual se generaron programas interdisciplinarios destacando la política de promoción y desarrollo de Espacios de Formación Integral (IFE) en todas las áreas de conocimiento, con resultados enriquecedores en los procesos formativos, de investigación y el diálogo entre universidad y sociedad. Similar situación desvela Quijije, D y Guzmán, A. (2017) en el Ecuador que tiene una política pública denominada El Programa Nacional de Extensión Universitaria desde el 2004 que señala y orienta la labor que deben cumplir las universidades pero que resulta insuficiente. Para el caso Colombia: Avendaño, W., Paz, L. y Rueda, G. (2017) señalan la importancia de la participación de la sociedad civil, los grupos de interés y los actores beneficiarios en la construcción de la Política Pública, de esta manera se aleja la idea que estas son respuestas 
unilaterales a los problemas sociales desde el Estado. Así la política Pública de educación superior de Colombia presenta características y articulación con otros países como Chile y México con la internacionalización, la inversión pública y la sostenibilidad financiera. Para el caso boliviano, Quiroga, L. y Rioja, G. (2017) señalan que no existen políticas serias de cambio en la gestión de extensión universitaria, del mismo modo las funciones de extensión se encuentran aisladas de la acción formativa e investigativa lo que impide la generación de conocimientos desde la relación docentes - estudiante y Universidad sociedad lo que sigue siendo un reto para construir la interacción con el proceso educativo y su vinculación con el proceso de conocimiento de la realidad social para determinar sus necesidades y satisfacer las. En Argentina, Gezmet, S. y Sánchez, E. (2017) dan cuenta que las universidades públicas logran constituir el año 2008 la Red Nacional de Extensión Universitaria (REXUNI) que luego elaboró el Plan Estratégico 2012-2015 para las universidades argentinas sobre cuya base las universidades asociadas construyen sus políticas de extensión universitaria particular.

De otro lado se tiene estudios que dan cuenta de las experiencias de Extensión universitaria entre los que destacan: el artículo de Gómez, J. (2019) estudiando los desafíos de la evaluación institucional en las universidades de Argentina sobre la base de las evaluaciones efectuadas por el CONEAU argentino establece que sus resultados pueden constituir una herramienta eficaz de intervención institucional que promueva el desarrollo de políticas de extensión universitaria que asegure la calidad, la pertinencia y la construcción de conocimientos en forma conjunta con la comunidad sobre la base del diálogo. Por lo tanto, concluye en que la extensión universitaria es una dimensión muy importante cuya función debe ser planificada, gestionada, documentada y evaluada. Por su parte Trejos y Ayala (2018) analizaron el papel de las tres funciones fundamentales que cumplen las universidades en la ciudad de Santiago de Cali Colombia promueven en teoría la formación integral de sus estudiantes, sin embargo el rol social de las universidades se han visto limitadas por las características socio económicas del medio como: la corrupción, el clientelismo, la manipulación de masas, lo que los obliga a efectuar tareas poco trascendentes para la transformación de su medio y se han reducido a acciones de imagen institucional. Este trabajo, muestra la incidencia de la realidad en el trastocamiento de los propósitos misionales y compromiso social de la universidad. Jiménez y Pérez (2017) estudiando el papel de la extensión universitaria en Colombia 
confirman que "las condiciones sociales, políticas, económicas, establecen lo que ha sido, es y será la Extensión”. Así, la interacción entre territorio y universidad es fundamental para que la universidad cumpla su función social porque ella posibilita la generación, transferencia y divulgación del conocimiento promoviendo posibles transformaciones en su estructura socio-económica. Este trabajo contribuye a entender las ventajas de la gestión integral de las funciones académicas en conjunto y la contribución de la universidad a la sociedad vía la extensión universitaria.

A nivel nacional en el Perú, este objeto de investigación ha sido poco tratado, hay escasos trabajos que dan cuenta de las experiencias particulares de algunas universidades, propuestas para su mejora o destacar su importancia, tampoco hay trabajos que haya sistematizado en conjunto la experiencia nacional de esta tercera función sustantiva de la universidad.

\section{2.- ESTRATEGIAS METODOLÓGICAS O MATERIALES Y MÉTODOS.}

El estudio se orientó por el método científico como la vía de producción de conocimiento y como tradición metodológica que orienta la investigación para abordar con objetividad y acierto del objeto de estudio. Orientó el trabajo de recolección de la información el método etnográfico. Se triangularon encuesta con lista de cotejo y análisis documental y siguiendo pautas de hermenéutica para interpretar los contenidos de la documentación reunida virtualmente habiendo privilegiado: la revisión de PEIs (Planes Estratégicos Institucionales), Estatutos, Reglamentos de Proyección Social /extensión universitaria, Informes, entre otros, complementándose con una encuesta y entrevistas estructuradas a los directores o encargados de estas funciones. El marco teórico se fue construyendo a lo largo de la investigación.

La sistematización del estudio ha siguiendo las estrategias de análisis y de deducciones e inducciones constantes y alternas hasta obtener gráficos y matrices de análisis semántico y categorial para construir el presente informe. Todo esto fue asistido por programas digitalizados como el Atlas Ti para los procedimientos cualitativos y el SPSSV25 para los datos cuantitativos.

Los instrumentos de investigación fueron sometidos a la validez mediante expertos, que dieron su opinión favorable sobre el contenido y constructo de la variable y dimensiones en estudio. La consistencia entre la variable, dimensiones, indicadores e ítems son muy relevantes que obedecen a las teorías empleadas por los investigadores. 


\section{3.- RESULTADOS Y DISCUSION}

El estudio centró su preocupación en develar la forma de trabajo que tienen las universidades del Perú en la función sustantiva de proyección social lo que implica estudiar: los instrumentos, programas, líneas de intervención, estrategias de trabajo, así como su articulación con las otras funciones y la participación universitaria en la agenda local, nacional y /o internacional. En este orden de ideas se tiene:

\section{Sobre los Instrumentos de gestión de la proyección social}

Concordante a los nuevos tiempos la gestión universitaria debe de asumir su función basada en planeamiento estratégico, esto implica entonces tener instrumentos que posibiliten establecer procedimientos que aseguren los procesos y su evaluación, así se buscó conocer los tipos, su instancia de aprobación institucional, su tiempo de aprobación y vigencia obteniéndose los siguientes resultados cuantitativos:

Tabla 1: Resultados de la dimensión instrumentos de gestión

\begin{tabular}{|c|c|c|c|c|c|c|c|}
\hline \multirow{3}{*}{ Ítems } & \multirow{3}{*}{ Respuestas } & \multicolumn{4}{|c|}{ Tipo de universidad } & \multirow{2}{*}{\multicolumn{2}{|c|}{ Total }} \\
\hline & & \multicolumn{2}{|c|}{ Pública } & \multicolumn{2}{|c|}{ Privada } & & \\
\hline & & $\bar{f}$ & $\%$ & f & $\%$ & f & $\%$ \\
\hline $\begin{array}{l}\text { En mi universidad las } \\
\text { actividades de proyección social } \\
\text { o extensión universitaria se } \\
\text { orientan por }\end{array}$ & Reglamento & 10 & 76.9 & 3 & 23.1 & 13 & 100 \\
\hline \multirow{2}{*}{$\begin{array}{l}\text { Este instrumento administrativo } \\
\text { cuenta con la aprobación de: }\end{array}$} & $\begin{array}{l}\text { Consejo } \\
\text { universitario }\end{array}$ & 10 & 76.9 & 2 & 15.4 & 12 & 92.3 \\
\hline & $\begin{array}{l}\text { Vice Rectorado } \\
\text { correspondiente }\end{array}$ & & 0.0 & 1 & 7.7 & 1 & 7.7 \\
\hline \multirow{4}{*}{$\begin{array}{l}\text { Este instrumento administrativo } \\
\text { cuenta con la aprobación de: }\end{array}$} & 2015 & 3 & 23.1 & 0 & 0 & 3 & 23 \\
\hline & 2016 & 4 & 30.8 & 1 & 7.7 & 5 & 38.5 \\
\hline & 2017 & 1 & 7.7 & 1 & 7.7 & 2 & 15.4 \\
\hline & 2018 & 2 & 15.4 & 1 & 7.7 & 2 & 23.1 \\
\hline $\begin{array}{l}\text { La vigencia de este instrumento } \\
\text { es: }\end{array}$ & $\begin{array}{l}\text { Cada periodo } \\
\text { Rectoral }\end{array}$ & 10 & 76.9 & 3 & 23.1 & 13 & 100 \\
\hline
\end{tabular}

Fuente: Base de datos de los investigadores - SPSSV25

Como se puede apreciar el total de las universidades tanto públicas como privadas orientan la función de proyección social con un reglamento aprobado por sus respectivos Consejos Universitarios. Sus redacciones datan entre el 2015 y 2018, de ellas un $61.5 \%$ fueron aprobados entre el 2015 al 2016 quedando sin modificación hasta la actualidad y el 38.5\% modificaron su primera redacción es decir en el periodo de adecuación de las universidades a la nueva ley, esto se corrobora con la indagación cualitativa que da cuenta 
que muchas de ellas son casi copias de anteriores reglamentos de proyección social, algunas le adoptaron la nomenclatura de responsabilidad social universitaria (RSU) como nueva dirección u oficina. Todo ello motivó que posterior al proceso de licenciamiento se hagan modificaciones estatutarias para rediseñar sus estructuras organizativas y sus funciones.

Otros instrumentos que complementan son los registros de inscripción de los grupos de proyectos de proyección social, algunas universidades cuentan con un sistema digital que registra, acopia los informes y procesa la certificación final.

\section{Planes, programas y líneas de intervención social}

Los trabajos de proyección social en estas universidades carecen de planes concretos, se ejecutan siguiendo lineamientos de acuerdos institucionales o lineamientos de la gestión de turno, apenas dos universidades cuentan con un plan de proyección social una pública y la otra privada. Respecto a los programas y líneas de intervención social el $23.1 \%$ señalan que hacen trabajos con la comunidad, el $61.5 \%$ con instituciones educativas y el $15.4 \%$ con instituciones productivas, con actividades de extensión universitaria en un $53,8 \%$ y 46,2\% con actividades de extensión cultural.

Tabla 2: Resultados de la dimensión planes, programas y líneas de investigación social

\begin{tabular}{|c|c|c|c|c|c|c|c|}
\hline \multirow{3}{*}{ Ítems } & \multirow{3}{*}{ Respuestas } & \multicolumn{4}{|c|}{ Tipo de universidad } & \multirow{2}{*}{\multicolumn{2}{|c|}{ Total }} \\
\hline & & \multicolumn{2}{|c|}{ Pública } & \multicolumn{2}{|c|}{ Privada } & & \\
\hline & & f & $\%$ & f & $\%$ & f & $\%$ \\
\hline \multirow{3}{*}{$\begin{array}{l}\text { En mi universidad los trabajos } \\
\text { de proyección social se hacen } \\
\text { en cumplimiento a: }\end{array}$} & Plan de proyección social & 2 & 15.4 & 0 & 0.0 & 2 & 15.4 \\
\hline & Acuerdo institucional & 7 & 53.8 & 3 & 23.1 & 10 & 76.9 \\
\hline & $\begin{array}{l}\text { Decisiones del director en } \\
\text { turno }\end{array}$ & 1 & 7.7 & 0 & 0.0 & 1 & 7.7 \\
\hline \multirow{3}{*}{$\begin{array}{l}\text { Señale cuales son los: } \\
\text { Programas de Proyección } \\
\text { social en su universidad: }\end{array}$} & Trabajos con la comunidad & 3 & 23.1 & 0 & 0.0 & 3 & 23.1 \\
\hline & $\begin{array}{l}\text { Con las instituciones } \\
\text { educativas }\end{array}$ & 5 & 38.5 & 3 & 23.1 & 8 & 61.5 \\
\hline & $\begin{array}{l}\text { Con las instituciones } \\
\text { productivas }\end{array}$ & 2 & 15.4 & 0 & 0.0 & 2 & 15.4 \\
\hline \multirow{2}{*}{$\begin{array}{l}\text { Señale cuales las Líneas de } \\
\text { Intervención social que } \\
\text { trabajan en su universidad: }\end{array}$} & Extensión cultural & 5 & 38.5 & 1 & 7.7 & 6 & 46.2 \\
\hline & Extensión universitaria & 5 & 38.5 & 2 & 15.4 & 7 & 53.8 \\
\hline \multirow{3}{*}{$\begin{array}{l}\text { Cómo se previene el } \\
\text { asistencialismo y paternalismo } \\
\text { en los proyectos sociales en su } \\
\text { universidad: }\end{array}$} & Políticas concretas & 1 & 7.7 & 2 & 15.4 & 3 & 23.0 \\
\hline & Proyectos cogestión & 5 & 38.5 & 0 & 0.0 & 5 & 38.5 \\
\hline & Enseñarles a hacer gestión & 4 & 30.8 & 1 & 7.7 & 5 & 38.5 \\
\hline
\end{tabular}

Fuente: Base de datos de los investigadores - SPSSV25 
Respecto a las medidas que adoptan las universidades para prevenir el asistencialismo y paternalismo en los proyectos de intervención social el $23.5 \%$ afirman tener políticas concretas, sin embargo, no dicen cómo lo que fue constatado por la indagación cualitativa no se encontraron estas políticas documentalmente más que en la declaración de los entrevistados con argumentaciones de buena intención. Así mismo el 38.5\% consideran que esto se previene estructurando proyectos cogestionados entre la universidad y los beneficiarios y otro $38.5 \%$ al enseñarles a hacer gestión para la solución del problema.

\section{Estrategias, procedimientos y actividades de Proyección Social}

El $69.2 \%$ de universidades afirman focalizar su intervención social en comunidades vulnerables sobre la base de convenios de cooperación a largo plazo, sin embargo, es contradictorio porque las revisiones de convenios por lo general son de mediano plazo van de uno a dos años con carácter renovable. Un 30.8\% delas universidades aseveran que estos convenios son esporádicos. De otro lado, los proyectos son diseñados desde la óptica de los docentes y estudiantes proyectistas por lo tanto es ínfima la cocreación con los beneficiarios así lo confirma el $84.6 \%$ que señalan que los hacen entre alguna vez $(69.2 \%)$ y nunca $(15.4 \%)$. Por lo tanto, estos proyectos carecen de impacto social y no contribuye al desarrollo sostenible.

Tabla 3. Resultados de la dimensión estrategias, procedimientos y actividades de Proyección social

\begin{tabular}{|c|c|c|c|c|c|c|c|}
\hline \multirow{3}{*}{ Ítems } & \multirow{3}{*}{ Respuestas } & \multicolumn{4}{|c|}{ Tipo de universidad } & \multirow{2}{*}{\multicolumn{2}{|c|}{ Total }} \\
\hline & & \multicolumn{2}{|c|}{ Pública } & \multicolumn{2}{|c|}{ Privada } & & \\
\hline & & $\mathbf{f}$ & $\%$ & f & $\%$ & f & $\%$ \\
\hline \multirow{4}{*}{$\begin{array}{l}\text { Se cuenta con convenios de } \\
\text { cooperación a largo plazo con } \\
\text { comunidades vulnerables, a fin de } \\
\text { focalizar la intervención social. }\end{array}$} & Siempre & 6 & 46.2 & 3 & 23.1 & 9 & 69.2 \\
\hline & $\begin{array}{l}\text { Algunas } \\
\text { veces }\end{array}$ & 2 & 15.4 & 0 & 0.0 & 2 & 15.4 \\
\hline & Nunca & 2 & 15.4 & 0 & 0.0 & 2 & 15.4 \\
\hline & Sub Total & 10 & 76.9 & 3 & 23.1 & 13 & 100.0 \\
\hline \multirow{4}{*}{$\begin{array}{l}\text { Se diseñan y desarrollan proyectos } \\
\text { cocreados con comunidades } \\
\text { vulnerables. }\end{array}$} & Siempre & 1 & 7.7 & 1 & 7.7 & 2 & 15.4 \\
\hline & $\begin{array}{l}\text { Algunas } \\
\text { veces }\end{array}$ & 7 & 53.8 & 2 & 15.4 & 9 & 69.2 \\
\hline & Nunca & 2 & 15.4 & 0 & 0.0 & 2 & 15.4 \\
\hline & Sub Total & 10 & 76.9 & 3 & 23.1 & 13 & 100.0 \\
\hline \multirow{4}{*}{$\begin{array}{l}\text { Se diseñan y desarrollan proyectos } \\
\text { que tienen un impacto social } \\
\text { duradero en el desarrollo } \\
\text { sostenible. }\end{array}$} & Siempre & 1 & 7.7 & 1 & 7.7 & 2 & 15.4 \\
\hline & $\begin{array}{l}\text { Algunas } \\
\text { veces }\end{array}$ & 7 & 53.8 & 2 & 15.4 & 9 & 69.2 \\
\hline & Nunca & 2 & 15.4 & 0 & 0.0 & 2 & 15.4 \\
\hline & Sub Total & 10 & 76.9 & 3 & 23.1 & 13 & 100.0 \\
\hline
\end{tabular}




\begin{tabular}{|c|c|c|c|c|c|c|c|}
\hline \multirow{4}{*}{$\begin{array}{l}\text { Se cuenta con mecanismos de } \\
\text { promoción del emprendimiento } \\
\text { para la sostenibilidad financiera de } \\
\text { los proyectos sociales }\end{array}$} & Siempre & 0 & 0.0 & 3 & 23.1 & 3 & 23.1 \\
\hline & $\begin{array}{l}\text { Algunas } \\
\text { veces }\end{array}$ & 3 & 23.1 & 0 & 0.0 & 3 & 23.1 \\
\hline & Nunca & 7 & 53.8 & 0 & 0.0 & 7 & 53.8 \\
\hline & Sub Total & 10 & 76.9 & 3 & 23.1 & 13 & 100.0 \\
\hline \multirow{4}{*}{$\begin{array}{l}\text { Se evalúa el impacto y se } \\
\text { sistematiza los proyectos sociales } \\
\text { para la mejora continua y el } \\
\text { escalamiento }\end{array}$} & Siempre & 0 & 0.0 & 2 & 15.4 & 2 & 15.4 \\
\hline & $\begin{array}{l}\text { Algunas } \\
\text { veces }\end{array}$ & 8 & 61.5 & 1 & 7.7 & 9 & 69.2 \\
\hline & Nunca & 2 & 15.4 & 0 & 0.0 & 2 & 15.4 \\
\hline & Sub Total & 10 & 76.9 & 3 & 23.1 & 13 & 100.0 \\
\hline \multirow{4}{*}{$\begin{array}{l}\text { Se alienta la participación de } \\
\text { contrapartes locales en el diseño, } \\
\text { ejecución y evaluación de los } \\
\text { proyectos sociales }\end{array}$} & Siempre & 2 & 15.4 & & 7.7 & 3 & 23.1 \\
\hline & $\begin{array}{l}\text { Algunas } \\
\text { veces }\end{array}$ & 5 & 38.5 & 2 & 15.4 & 7 & 53.8 \\
\hline & Nunca & 3 & 23.1 & 0 & 0.0 & 3 & 23.1 \\
\hline & Sub Total & 10 & 76.9 & 3 & 23.1 & 13 & 100.0 \\
\hline
\end{tabular}

Fuente: Base de datos de los investigadores - SPSSV25

De otro lado es muy débil el desarrollado de mecanismos de promoción del emprendimiento para la sostenibilidad financiera de los proyectos sociales, estos son en su totalidad financiados por cada grupo de proyección social. Las universidades públicas cuentan con cierto financiamiento para la extensión cultural más no para la proyección social, mientras que en las particulares hay un ínfimo financiamiento que tiene que ver con la política de posicionamiento institucional. Así lo muestra la tabla en la el 53.8\% de universidades declaran no contar con mecanismos de promoción del emprendimiento para la sostenibilidad financiera de los proyectos sociales y el 23. $1 \%$ aseveran que reciben esporádicamente y siempre reciben el $23.1 \%$ que son precisamente universidades particulares, como es de notar ninguna pública. Los informes de los proyectos de intervención social tienen el carácter de fiscalización sobre el cumplimiento de lo programado y el gasto efectuado por el grupo para la certificación correspondiente.

La evaluación del impacto y la sistematización los proyectos sociales deben servir para la mejora continua y el escalamiento, asunto que el 69.2\% de universidades aseveran que lo hacen algunas veces, destacando en ellas un $61.5 \%$ de universidades que son públicas, mientras que el $15.4 \%$ que afirman hacerlo siempre son privadas.

En la revisión de actas, informes y revistas de las direcciones de proyección social se han encontrado algunos acuerdos y declaraciones sueltas para alentar la participación de contrapartes locales o denominados también como grupos de interés en el diseño, ejecución y evaluación de los proyectos sociales, pero no se han concretado en una 
política institucional universitaria, lo que queda refrendada con lo que la tabla especifica que el $53.8 \%$ de las universidades lo hacen algunas veces.

\section{Articulación de Proyección Social con Formación e Investigación}

Los procesos de articulación entre formación profesional, investigación y extensión social como funciones sustanciales de la universidad es muy débil. Cada una de estas funciones se desarrollan por separado buscando destacar cada una a su manera. La gestión universitaria en estos últimos tiempos de pos-licenciamiento y acreditación le ha dado mayor apoyo a la investigación para alcanzar los estándares, seguido de la parte formativa, mientras que la proyección social se encuentra postergada. Lo óptimo sería que debieran existir mecanismos para que los proyectos sociales y las investigaciones contribuyan en conjunto a la mejora de los contenidos de la formación profesional y la investigación. Véase la siguiente tabla:

Tabla 4: Resultados de la dimensión articulación de proyección social con formación e investigación

\begin{tabular}{|c|c|c|c|c|c|c|c|}
\hline \multirow[b]{3}{*}{ Ítems } & \multirow[b]{3}{*}{ Respuestas } & \multicolumn{4}{|c|}{ Tipo de universidad } & \multirow{2}{*}{\multicolumn{2}{|c|}{ Total }} \\
\hline & & \multicolumn{2}{|c|}{ Pública } & \multicolumn{2}{|c|}{ Privada } & & \\
\hline & & f & $\%$ & $\mathbf{f}$ & $\%$ & f & $\%$ \\
\hline \multirow{4}{*}{$\begin{array}{l}\text { Se desarrollan procesos de } \\
\text { articulación entre formación } \\
\text { profesional, investigación y } \\
\text { extensión social solidaria. }\end{array}$} & Siempre & 0 & 0.0 & 2 & 15.4 & 2 & 15.4 \\
\hline & $\begin{array}{l}\text { Algunas } \\
\text { veces }\end{array}$ & 4 & 30.8 & 1 & 7.7 & 5 & 38.5 \\
\hline & Nunca & 6 & 46.2 & 0 & 0.0 & 6 & 46.1 \\
\hline & Sub Total & 10 & 76.9 & 3 & 23.1 & 13 & 100 \\
\hline Existen mecanismos para & Siempre & 0 & 0.0 & 1 & 7.7 & 1 & 7.7 \\
\hline $\begin{array}{l}\text { que los proyectos sociales y } \\
\text { las investigaciones en }\end{array}$ & $\begin{array}{l}\text { Algunas } \\
\text { veces }\end{array}$ & 1 & 7.7 & 2 & 15.4 & 3 & 23.1 \\
\hline $\begin{array}{l}\text { comunidad contribuyan en la } \\
\text { mejora de los contenidos de }\end{array}$ & Nunca & 9 & 69.2 & 0 & 0.0 & 9 & 62.2 \\
\hline $\begin{array}{l}\text { la formación profesional y la } \\
\text { investigación }\end{array}$ & Sub Total & 10 & 76.9 & 3 & 23.1 & 13 & 100 \\
\hline
\end{tabular}

Fuente: Base de datos de los investigadores - SPSSV25

Solo $15.4 \%$ de las universidades afirman que tienen procesos de articulación, frente a un $46.1 \%$ que señalan no tener esta relación entre las tres funciones fundamentales de la universidad, y un 38,5\% que refiere hacerlo algunas veces.

Como consecuencia de lo anotado se evidencia que no existen mecanismos institucionales para que los proyectos sociales y las investigaciones en comunidad contribuyan en la mejora de los contenidos de la formación profesional y la investigación, así el $62.2 \%$ 
menciona que nunca de establecido, el $23.1 \%$ dice que lo hacen algunas veces y apenas el 7.7\% afirma hacerlo siempre correspondiendo esta afirmación a la universidad privada.

\section{Participación en la agenda pública local, nacional y/o internacional}

Desde los albores de la universidad como institución adquirió una relación estrecha con la sociedad y siempre fue centro de irradiación de pensamiento. Su opinión y acción fue respetado por las otras instituciones. La palabra de la academia siempre era considerada como una buena fuente de opinión ante cualquier preocupación colectiva concordante con el tiempo y el proceso histórico.

Véase lo que sucede en la actualidad, así se tiene que las altas autoridades de la universidad en un $76.9 \%$ trabajan algunas veces en equipo para potenciar el rol social de la universidad, mientras que un 15.4 refieren que no lo hacen nunca y apenas un $7,7 \%$ aseguran que siempre lo hacen. Esto es fortalecido por los datos cualitativos que muestran interferencias en la gestión universitaria por razones de orden político, acceso a los financiamientos y celos profesionales entre sectores profesionales y desencuentros en el logro de objetivos institucionales según manifiestan sus actores sociales.

Tabla 5: Resultados de la dimensión participación en la agenda pública: Local, nacional y/o internacional

\begin{tabular}{|c|c|c|c|c|c|c|c|}
\hline \multirow[b]{3}{*}{ Ítems } & \multirow[b]{3}{*}{ Respuestas } & \multicolumn{4}{|c|}{ Tipo de universidad } & \multirow{2}{*}{\multicolumn{2}{|c|}{ Total }} \\
\hline & & \multicolumn{2}{|c|}{ Pública } & \multicolumn{2}{|c|}{ Privada } & & \\
\hline & & f & $\%$ & $\mathbf{f}$ & $\%$ & f & $\%$ \\
\hline \multirow{4}{*}{$\begin{array}{l}\text { Las altas autoridades de la } \\
\text { universidad trabajan en equipo } \\
\text { para potenciar el rol social de } \\
\text { la universidad. }\end{array}$} & Siempre & 0 & 0.0 & 1 & 7.7 & 1 & 7.7 \\
\hline & $\begin{array}{l}\text { Algunas } \\
\text { veces }\end{array}$ & 8 & 61.5 & 2 & 15.4 & 10 & 76.9 \\
\hline & Nunca & 2 & 15.4 & 0 & 0.0 & 2 & 15.4 \\
\hline & Sub Total & 10 & 76.9 & 3 & 23.1 & 13 & 100.0 \\
\hline \multirow{4}{*}{$\begin{array}{l}\text { Hacen pronunciamientos } \\
\text { sobre la problemática socio } \\
\text { ambiental: local, regional o } \\
\text { nacional. }\end{array}$} & Siempre & 0 & 0.0 & 0 & 0.0 & 0 & 0.0 \\
\hline & $\begin{array}{l}\text { Algunas } \\
\text { veces }\end{array}$ & 2 & 15.4 & 3 & 23.1 & 5 & 38.5 \\
\hline & Nunca & 8 & 61.5 & 0 & 0.0 & 8 & 61.5 \\
\hline & Sub Total & 10 & 76.9 & 3 & 23.1 & 13 & 100.0 \\
\hline \multirow{4}{*}{$\begin{array}{l}\text { Se cuenta con convenios de } \\
\text { cooperación con actores } \\
\text { públicos y privados para la } \\
\text { agenda ODS- } 2030\end{array}$} & Siempre & 1 & 7.7 & 0 & 0.0 & 1 & 7.7 \\
\hline & $\begin{array}{l}\text { Algunas } \\
\text { veces }\end{array}$ & 4 & 30.8 & 3 & 23.1 & 7 & 53.8 \\
\hline & Nunca & 5 & 38.5 & 0 & 0.0 & 5 & 38.5 \\
\hline & Sub Total & 10 & 76.9 & 3 & 23.1 & 13 & 100.0 \\
\hline
\end{tabular}




\begin{tabular}{|c|c|c|c|c|c|c|c|}
\hline Se genera procesos de & Siempre & 2 & 15.4 & 1 & 7.7 & 3 & 23.1 \\
\hline $\begin{array}{l}\text { participación e incidencia de la } \\
\text { comunidad universitaria en la }\end{array}$ & $\begin{array}{l}\text { Algunas } \\
\text { veces }\end{array}$ & 3 & 23.1 & 2 & 15.4 & 5 & 38.5 \\
\hline agenda ODS a nivel local, & Nunca & 5 & 38.5 & 0 & 0.0 & 5 & 38.5 \\
\hline nacional e internacional & Sub Total & 10 & 76.9 & 3 & 23.1 & 13 & 100.0 \\
\hline universidad tiene & Siempre & 0 & 0.0 & 3 & 23.1 & 3 & 23.1 \\
\hline $\begin{array}{l}\text { incidencia en la discusión y el } \\
\text { diseño de políticas públicas de }\end{array}$ & $\begin{array}{l}\text { Algunas } \\
\text { veces }\end{array}$ & 5 & 38.5 & 0 & 0.0 & 5 & 38.5 \\
\hline desarrollo local, regional o & Nunca & 5 & 38.5 & 0 & 0.0 & 5 & 38.5 \\
\hline nacional. & Sub Total & 10 & 76.9 & 3 & 23.1 & 13 & 100.0 \\
\hline La universidad mantiene & Siempre & 0 & 0.0 & 0 & 0.0 & 0 & 0.0 \\
\hline $\begin{array}{lrrr}\text { diálogo permanente } & \text { y } & \text { de } \\
\text { cooperación con } & & \text { las }\end{array}$ & $\begin{array}{l}\text { Algunas } \\
\text { veces }\end{array}$ & 3 & 23.1 & 3 & 23.1 & 6 & 46.2 \\
\hline instituciones públicas $\mathrm{y}$ & Nunca & 7 & 53.8 & 0 & 0.0 & 7 & 53.8 \\
\hline privadas. & Sub Total & 10 & 76.9 & 3 & 23.1 & 13 & 100.0 \\
\hline
\end{tabular}

Fuente: Base de datos de los investigadores - SPSSV25

Frente a la apremiante problemática socio ambiental: local, regional o nacional, el $61.5 \%$ de las universidades afirman contundentemente que nunca se pronuncian, y el $38.5 \%$ lo hacen algunas veces, lo que es comprobado por los datos cualitativos y se respalda en pronunciamientos esporádicos de los Consejos universitarios frente a problemas graves a las que han respondido por la presión de los estudiantes y sindicatos universitarios, más no como institución sensible de la sociedad.

Frente a la agenda internacional que los países del mundo asisten frente a la inviabilidad del sistema socio ambiental y los acuerdos internacionales como la Agenda ODS-2030 en la están comprometidos los estados e instituciones sociales del mundo la universidad peruana debería contar convenios de cooperación con actores públicos y privados para el logro de sus 17 objetivos, la tabla 5 muestra que 53. 8\% de universidades tienen algunos convenios de cooperación con actores públicos y privados para trabajar la agenda ODS2030, mientras que el $38.5 \%$ refieren que a la fecha no lo han efectuado, solo el7.7\% afirman haber suscrito estos convenios. En esa misma lógica la universidad debiera genera procesos de participación e incidencia de la comunidad universitaria en la agenda ODS-2030 a nivel local, nacional e internacional, sin embargo, la tabla muestra que el $77.0 \%$ de universidades han generado estos procesos de participación entre algunas veces y nunca divididos en $38.5 \%$ cada uno respectivamente. Los datos confirman ya que se 
han visto proyectos con este compromiso, pero al interior de las universidades y muy escaso para el externo.

En cuanto a que, si la universidad tiene incidencia en la discusión y el diseño de políticas públicas de desarrollo local, regional o nacional, el 38.5\% de universidades aseveran que intervienen algunas veces, un $23.1 \%$ declaran hacerlo siempre frente a un $38.5 \%$ que señalan que nunca lo hacen. Lo anterior se complementa finalmente con que, si la universidad mantiene diálogo permanente y de cooperación con las instituciones públicas y privadas, los datos cuantitativos muestran que el 53.8\% de universidades no tienen estas relaciones de manera permanente, mientras que en el $46.2 \%$ esta relación se efectúa ocasionalmente, lo que queda refrendada por la indagación cualitativa a través de los convenios interinstitucionales que se trató líneas arriba.

\section{DISCUSIÓN}

Los hallazgos del presente estudio muestran que la gestión de la proyección social/ extensión universitaria en el Perú no cuenta con políticas concretas, como si lo hacen otros países de América Latina como: Ecuador que tiene una política pública denominada Programa Nacional de Extensión Universitaria desde el 2004 tal como lo muestran Quijije, D. y Guzmán, A.(2017), Argentina que tiene el Plan estratégico 2012-2015 Gezmet, S. y Sánchez, E. (2017) sobre cuya base se estructural las políticas particulares de cada universidad; del mismo, modo Avendaño, W., Paz, L. y Rueda, G. (2017) confirman que Colombia también tiene política pública de extensión en su ley de educación nacional, destacando la participación de los beneficiarios de la sociedad civil y los grupos de interés en su construcción.

En el Perú esto todavía está ausente desde el propio estado nacional dejando a la libre acción de cada universidad en base de su supuesta autonomía.

Esta situación ha generado en la universidad peruana:

1) Un aislamiento de la universidad frente a la problemática local-regional, nacionalmundial de manera que al no involucrarse en su dinámica cotidiana la universidad renuncia su carácter histórico de compromiso social para el desarrollo y la transformación, habiéndose reducido a la formación y calificación de cuadros técnico profesionales para el sistema empujados por la lógica hegemónica del sistema mundo.

2) Un trabajo interno también separado entre las funciones sustantivas de la universidad, donde cada una resuelve sus necesidades y promociona sus logros, no garantiza una 
formación integral del futuro profesional ciudadano.

3) Una desigual valoración de las funciones de investigación, formación y extensión tanto en el producto de su trabajo como en la asignación presupuestal, donde la tercera función es el menos tomada en cuenta.

4) La subvaluación de la función de la proyección social/ extensión universitaria genera que las otras funciones fundamentales o sustantivas de la universidad no cumplan cabalmente sus tareas y objetivos estratégicos.

5) Generación de proyectos de intervención social asistemáticos, caracterizados por ser: voluntaristas, asistencialistas, extracurriculares, dispersos y desfinanciados. Frente a ello no se han generado mecanismos para evitarlos o superarlos, se sigue viendo a la población objetivo de la proyección social como los beneficiarios, interesados que solo esperan ayudas o favores de la universidad.

Los estudios de Quiroga, L. y Rioja, G. (2017) realizados en las universidades de Bolivia muestran que tampoco cuentan con políticas de gestión de extensión universitaria coincidiendo con la presente exposición.

En este tiempo de profundos cambios socio-económicos una buena manera de superar estas limitaciones de la proyección social en las universidades del Perú será:

1) Comprometiéndose y asumiendo la Agenda 2030 y sus 17 objetivos, ello permitirá alinear las acciones de proyección social de cada facultad, de este modo se puede contribuir a la superación de los problemas sociales de las poblaciones en vulnerabilidad. A su vez permitirá diseñar las áreas y líneas de intervención social para posibilitar un trabajo inter, trans y multidisciplinario en la solución de problemas urgentes de la demanda territorial con innovaciones social y ambientalmente responsables.

2) Articular las tres funciones sustantivas de la universidad: formación, investigación y proyección social en los distintos momentos del proyecto de intervención social, esto permitirá retroalimentar a cada una de las funciones: poniendo en práctica los conocimientos, debatiéndolos in situm, recogiendo conocimientos de los beneficiarios, poniendo en práctica los procesos de investigación para la intervención y finalmente buscando una solución creativa a las demandas. Al final los proyectos de intervención podrán recomendar nuevos temas y líneas para la investigación y para las estructuras curriculares más allá del logro de sus objetivos sociales. 
3) Promover la participación directa de los grupos beneficiarios en la proyección social, en condición de co creadores y co gestores desde el momento del diseño del proyecto, la gestión y la sistematización. El trabajo conjunto hará que cambien de actitud frente a la universidad y sus responsabilidades. Dejaran de ser asistidos y pasaran a tener protagonismo en los trabajos donde en igualdad de condiciones podrán dialogar y buscar soluciones conjuntas, de este modo en éste dialogo de saberes gana la población beneficiaria y gana la universidad por lo que se alcanzaría mayor impacto en la intervención social.

4) Capacitación en métodos del trabajo social universitario, solo un trabajo planificado, con metodologías adecuadas pueden tener resultados óptimos y de impacto. Se requiere que los docentes y estudiantes de proyección social tengan que poner en práctica cuando menos los siguientes métodos: la Investigación - acción (I-A) y el de aprendizaje - Servicio $(\mathrm{A}+\mathrm{S})$.

5) Comprometiendo la participación de la universidad en la Agenda pública, por su naturaleza la universidad no pude retraerse a su recinto, al contrario, con la lucidez de sus investigaciones y los conocimientos que maneja académicamente debe de estar presente en el diseño, debate y ejecución de las políticas públicas del territorio de su influencia: local, regional y nacional.

6) Comprometer un presupuesto para proyectos de intervención social, es necesario incentivar la creatividad, en todas sus modalidades, esta vez en la búsqueda de soluciones a problemas socio ambientales que propicien una mejor calidad de vida. Este presupuesto debe ser concursable, de este modo los proyectos serán más responsables y tendrán mayor impacto.

Finalmente, la adopción de la RSU como paradigma de la gestión universitaria no restringe la función la proyección social sino al contrario la potencia con beneficiosos resultados para el logro eficaz del compromiso social de la universidad con la sociedad. Para ello hay necesidad de contar con políticas públicas e institucionales que renueven el trabajo de esta sustantiva función devaluada y postergada y perjudica la institucionalidad universitaria.

\section{4.- CONCLUSIÓN O CONSIDERACIONESFINALES}

1) La Proyección Social como la tercera función fundamental en las universidades del Perú no cuentan con políticas públicas específicas, como si lo tienen otros países entre 
las que destacan Ecuador, Argentina, Colombia en América Latina. Su función se encuentra señalada a grandes rasgos en la Ley universitaria $N^{\circ} 30220$ dejando a cada universidad la potestad de diseñar sus propias políticas institucionales basadas en su autonomía. Sin embargo, sus reglamentos no responden a los retos del contexto mundial y se confunde con la Responsabilidad Social Universitaria (RSU), llegando a la simplicidad de sustituir de nombre por ésta última. Hay una imprecisión del papel de la proyección social universitaria en el nuevo contexto debido al incorrecto entendimiento de la RSU en el desarrollo institucional. Las políticas que se tienen en las universidades públicas y privadas son disimiles e imprecisas en su papel como fuente de desarrollo social y cultural. No hay un entendimiento único de lo que es la proyección social, surgiendo con ella: asistencialismo, paternalismo, filantropía; caracterizada por ser voluntarista, asistencialista, extracurricular, dispersos y desfinanciados.

2) La ausencia de políticas de proyección social ha propiciado una débil articulación con los procesos de formación e investigación. Cada proceso tiene una dinámica aislada de las otras lo que dificulta la formación integral del estudiante e interfiere el cumplimiento del compromiso histórico de la universidad con la sociedad. En esta relación la función de proyección social resulta devaluada al considerarse como tareas: "secundarias", "complementarias", "actividades informales", "residuales" o "poco trascendentes", desconociendo con ello el papel de relacionante directo con la sociedad y su problemática, por lo mismo la universidad no participa en la agenda pública y no puede promover el desarrollo territorial de su ámbito de influencia.

3) La función de proyección social /extensión universitaria en el Perú al no contar con políticas renovadas a la luz de los retos de la sociedad global mantienen viejos modelos organizacionales con funciones que impiden propiciar la participación directa de los grupos beneficiarios en la labor de proyección social, en condición de co creadores y co gestores y hacer que las intervenciones sociales tengan sostenibilidad.

\section{5.- LISTA DE REFERENCIAS}

Arocena, R, Tommasino, H., Rodriguez, N., Sutz, J., Pedrosian, E. Á., \& Romano, A. (2017). Integralidad: tensiones y perspectivas. Cuadernos de Extensión № 1 . Año 1. Montevideo. CSEAM. 
Avendaño Castro, William Rodrigo; Paz Montes, Luisa; Rueda Vera, Gerson (2017) Políticas públicas y educación superior: análisis conceptual del contexto colombiano. Revista Venezolana de Gerencia, vol. 22, núm. 79, 2017, Universidad del Zulia, Venezuela.

ANR Asamblea Nacional de Rectores (2007) Definición y alcances de la Extensión Universitaria y Proyección Social en la Universidades del País. Lima Perú.

Bourdieu, P. (2001) Las estructuras sociales de la economía. Editorial Manantial, Buenos Aires Argentina.

Cano, Agustín y Castro, Diego (2016) La extensión universitaria en la transformación de la educación superior. El caso de Uruguay. En Revista Andamios Volumen 13, número 31, mayo-agosto, 2016, pp. 313-337.

CONEAU (2008) Consejo de Evaluación, Acreditación y Certificación de la Calidad de la Educación Superior Universitaria. Lima Perú

D’Andrea, R. E., Zubiría y Sastre Vázquez (s/f.), Reseña histórica de la extensión universitaria. Universidad Argentina.

Fernández Lamarra, Norberto; Coppola, Natalia. (2014). La reforma del 18 y la autonomía universitaria. En: Teodoro, Antonio; Beltrán, José (Comp.). Sumando voces. Ensayos sobre Educación Superior en términos de igualdad e inclusión social. Miño y Dávila Editores, Buenos Aires, Argentina.

Gargantini, D. (2014). Estado de la Responsabilidad Social Universitaria - El aporte de AUSJAL a la institucionalización en América Latina. En Cuaderno de RSO. Publicación interdisciplinaria sobre responsabilidad social de las organizaciones. 2 (1), 17-35.

Gezmet, Sandra y Sánchez, Esther. (2017) Debates actuales sobre extensión universitaria. En Asignatura Extensión Universitaria, compendio bibliográfico. Barrientos, M. (Compilador) Universidad Nacional de Córdova, Secretaría de extensión Universitaria. Córdova Argentina.

Gómez Arn, Johana. N. (2019). Desafíos de la evaluación institucional en el aseguramiento de la calidad de la extensión universitaria. RAES, 11(18), pp. 136149.

Habermar, J. (1987) Teoría de la acción comunicativa, Taurus.4ta ed., Madrid España. 
Jiménez, A. y Pérez, J. (2017) Universidad y territorio. Las misiones: una estrategia de Extensión Universitaria con sentido territorial. Colombia. Kénosis/Vol. 5/n. ${ }^{\circ} 8$ /pp. 118-143.

Méndez, Gustavo (2018) Desarrollo y conceptualización de la Extensión Universitaria: Un aporte de la Secretaría de Extensión de la Universidad Nacional del Litoral para al análisis y debate acerca de la Extensión Universitaria. Secretaría de Extensión de la Universidad Nacional del Litoral (UNL), Santa Fe Argentina.

Mintzberg, H. y Jorgensen, J. (1995) Una estrategia emergente para la política pública. Rev. Gestión y Política Pública, vol IV, num 1, primer semestre. INAP, Madrid: $25-46$.

Miranda-Suárez, Efraín F. (2010). La Proyección Social en los cincuenta años de la Facultad de Sociología de la UNCP. Revista Universidad Nacional del Centro del Perú.

Miranda-Suárez, Efraín F. (2019) Responsabilidad Social universitaria: una visión desde las universidades de la macro región centro del Perú. Tesis Doctoral, Universidad Nacional del centro del Perú, Unidad de Posgrado de la facultad de Educación, Huancayo Perú.

Núñez, A., Álvarez, B. y Martínez, C. (2017) La extensión universitaria y su relación con la formación inicial de las carreras pedagógicas en Cuba. En Revista Electrónica “Actualidades Investigativas en Educación”, Universidad de Costa Rica, Facultad de Educación, Instituto de Investigación en Educación (INIE). Costa Rica.

Quijije, Diómedes y Guzmán, Antonio (2017) Extensión universitaria: Reflexiones de la urgencia de perfeccionar su accionar. Revista Electrónica Formación y Calidad Educativa (REFCalE) Vicerrectorado Académico de la Universidad Laica Eloy Alfaro, Manabí, Ecuador.

Quiroga Moreno, Luis y Rioja Montaño, Greby U. (2017) La Universidad y los Procesos de Integración Social La interacción social universitaria: de la prestación de servicios al proceso pedagógico de la educación superior. Página electrónica Research GATE. 
Santos, B. D. S. (2012). De las dualidades a las ecologías. Serie: Cuaderno de Trabajo No 18. La Paz. Bolivia. Red Boliviana de Mujeres Transformando la Economía, REMTE.

Trejos, J. y Ayala, J. (2018) Integración de las funciones sustantivas de la educación Superior: un aporte para la construcción de paz. Tesis de maestría, Santiago de Cali Colombia. Pontificia Universidad Javeriana Facultad de Humanidades y Ciencias Sociales.

Tünnermann, Carlos (2002). El nuevo concepto de la Extensión Universitaria; Cuaderno № 5, Universidad de Carabobo. Venezuela, 2002.

Tünnermann, Carlos (2008). La Reforma de Córdoba. Vientre fecundo de la transformación universitaria. En La reforma universitaria: desafíos y perspectivas noventa años después (págs. 16-19). Buenos Aires: CLACSO.

UNESCO. Comunicado de la Conferencia mundial de educación superior (2009) Las Nuevas Dinámicas de la Educación Superior y de la Investigación para el Cambio Social y el Desarrollo. UNESCO, París, 5-8 de julio de 2009.

Vallaeys, François. (2016) Responsabilidad Social Universitaria: Un Modelo ÉticoPolítico Para Reformar La Universidad En El Perú. Nota de política N6, Escuela de Gestión Pública, Universidad del Pacífico, Lima Perú.

Vallaeys, François (2018) Investigación continental de autodiagnóstico RSU: Avances institucionales en 12 metas socialmente responsables. URSULA, CAF, Universidad del Pacífico, Lima Perú.

Vallaeys, François. (2019) Responsabilidad Social Universitaria: Un Modelo ÉticoPolítico para Reformar la Universidad en el Perú. Lima Perú, Escuela de Gestión Pública (EGP), Universidad del Pacífico. 